\title{
A gas-accepting ion source for Accelerator Mass Spectrometry: Progress and Applications
}

\author{
M.L. Roberts, K.F. von Reden, J.R. Burton, C.P. McIntyre, and S.R. Beaupré \\ Woods Hole Oceanographic Institution, Woods Hole, MA 02543 USA
}

\begin{abstract}
The National Ocean Sciences Accelerator Mass Spectrometry (NOSAMS) facility at the Woods Hole Oceanographic Institution has developed an Accelerator Mass Spectrometry (AMS) system designed specifically for the analysis of ${ }^{14} \mathrm{C}$ in a continuously flowing stream of carrier gas. A key part of the system is a gas-accepting microwave ion source. Recently, substantial progress has been made in the development of this source, having achieved ion currents rivaling that of a traditional graphite source (albeit at relatively low efficiency). Details and present performance of the gas source are given. Additionally, representative results obtained from coupling the source to both a gas chromatograph and gas bench are presented.
\end{abstract}

\section{Introduction}

In traditional AMS, ion beams are produced by sputtering from prepared graphite targets. While converting $\mathrm{CO}_{2}$ to graphite is a well-established process ${ }^{[1]}$, the necessary steps are timeand labor-intensive. It would be ideal, if one could skip the graphitization process and inject $\mathrm{CO}_{2}$ directly into an ion source. To that end, a 'hybrid' gas ion source can be purchased from either of the two major manufactures of AMS systems (i.e., National Electrostatics Corporation, Middleton, WI, USA and High Voltage Engineering Europa B.V, Amersfoort, Netherlands). In a 
hybrid gas ion source, $\mathrm{CO}_{2}$ is directed to and adsorbed by a titanium pellet from which $\mathrm{C}^{-}$ions are extracted via cesium sputtering. The disadvantage of a hybrid gas ion source is that the titanium pellet needs to be changed between samples to avoid sample-to-sample memory ${ }^{[2]}$ and, when compared to a traditional graphite ion source, it produces relatively low ion currents.

Additionally, if one wishes to couple a gas-chromatograph or a high-pressure liquidchromatograph to a hybrid gas ion source, it becomes necessary to change titanium pellets in the time interval between peaks in order to determine the precise ${ }^{14} \mathrm{C}$ content of each chromatographic peak. Knowing when to change pellets is difficult without prior knowledge of both the chemical structure of the sample and the volatility of its individual components. Also, in many cases, the difference in the ${ }^{14} \mathrm{C}$ content of chromatographic peaks can be small and high-

currents (i.e., high ${ }^{14} \mathrm{C}$ counting rates) are needed to achieve precise results. Simply put, there is a poor match between the methods of analytical organic chemistry and the operational capabilities of a hybrid gas ion source. A high-current, low-memory, gas-accepting ion source is needed.

\section{The Gas Ion Source}

The operating principle of the gas ion source is outlined in Figure $1 . \mathrm{CO}_{2}$ is introduced (with or without Argon carrier gas) into a plasma chamber using a fused-silica capillary. By varying the length of the capillary, different gas flow rates are realized. A plasma is established (at or near the 'Electron Cyclotron Resonance' condition) using $2.45 \mathrm{GHz}$ microwaves and an axial magnetic field. Positive ions (several $\mathrm{mA}$ ) are extracted from the plasma using a triode arrangement consisting of the plasma chamber, an acceleration electrode, and an electron suppression (or deceleration) electrode. Negative ions are formed by charge-exchanging 
interactions with magnesium. The resulting ion beam is characterized by a fairly large emittance ( $\sim 100 \pi \mathrm{mm}$ mrad) and a large energy spread $(\sim \pm 325 \mathrm{eV})$. Details of the gas ion source can be found elsewhere ${ }^{[3]}$. For high efficiency transmission of the beam, the low-energy end of the AMS system was specifically designed to be achromatic and have a large acceptance ${ }^{[4,5]}$. Additionally, the rest of the AMS system (i.e., the accelerator and the high-energy achromat) was also designed to have large acceptance ${ }^{[4]}$.

Table 1 lists ion source output, source efficiency, various transmission efficiencies, and total system efficiency. Measurements were made with the ion source operating at $40 \mathrm{kV}$ with respect to ground. Maximum ion source output was obtained with a methyl-deactivated, fusedsilica, capillary (SGE Analytical Science) of 100- $\mu \mathrm{m}$ inner diameter and approximate $1.6 \mathrm{~m}$ length corresponding to a $\mathrm{CO}_{2}$ flow rate of $370 \mu \mathrm{l} / \mathrm{min}$. Optimal operating temperature of the charge exchange canal was about $445{ }^{\circ} \mathrm{C}$. Total system efficiency was determined using the known $\mathrm{CO}_{2}$ flow rate and known ${ }^{14} \mathrm{C}$ content. In our case, total efficiency is defined as the proportion of ${ }^{14} \mathrm{C}$ ions detected to the number of $\mathrm{CO}_{2}$ ions introduced into the source. Within the range of flow rates tested (100 to $600 \mu \mathrm{l} / \mathrm{min}$ ), a weak inverse-correlation was found between system efficiency and $\mathrm{CO}_{2}$ flow rate (i.e., lower flow rates gave higher efficiency).

Source 'memory' could be divided into a short- and long-term component. Source memory was determined by alternately injecting 'live' and 'dead' $\mathrm{CO}_{2}$ into the ion source. The Fraction Modern $\left(\mathrm{F}_{\mathrm{m}}\right)$ of the 'live' and 'dead' $\mathrm{CO}_{2}$ was approximately 1.0438 and 0.003 respectively (as determined by conventional AMS). After running 'live' $\mathrm{CO}_{2}$, and upon switching to 'dead' $\mathrm{CO}_{2}$, the short-term memory component was characterized by the measured 
${ }^{14} \mathrm{C} /{ }^{12} \mathrm{C}$ rate quickly dropping to a level of $\mathrm{F}_{\mathrm{m}} \approx 0.010$ (equivalent to a Libby age of $\approx 37,000$ yrs.) with an exponential time constant of approximately $2 \mathrm{~s}$. This short-term memory can be explained by gas mixing/flushing within the approximately $100 \mathrm{ml}$ plasma chamber. A long-term component to the memory was also seen. After quickly dropping to $\mathrm{F}_{\mathrm{m}} \approx 0.010$, the measured ${ }^{14} \mathrm{C} /{ }^{12} \mathrm{C}$ rate dropped slowly over the next few minutes to a base level of $\mathrm{Fm} \approx 0.005$ (equivalent to a Libby age of $\approx 42,000$ yrs.). Various tests indicated a correlation between gas flow rate and memory (i.e., a shorter capillary, giving a higher flow rate, showed less memory). To see if this was a surface effect from the capillary walls, both larger and smaller inner diameter capillaries were compared to the standard 100- $\mu \mathrm{m}$ inner diameter, $1.6 \mathrm{~m}$ long capillary $\left(\mathrm{CO}_{2}\right.$ flow rate 370 $\mu \mathrm{l} / \mathrm{min}$ ). To keep the flow rates the same for comparison purposes, the length of each of the various diameter capillaries was adjusted. In all cases, the long-term memory didn't appreciably change between differing diameter capillaries. The cause of the long-term memory, and why it varies with overall gas flow rate, is not fully understood and is currently under investigation.

\section{Gas-Bench}

We have recently developed a 'gas-bench' type interface for the ion source that allows rapid, low-cost measurements of carbonaceous type samples (e.g., corals, carbonaceous sediments, etc.). The process begins by weighing $25-30 \mathrm{mg}$ of pre-cleaned sample material into a $7 \mathrm{~mL}$ vacutainer. The vacutainer is then evacuated to approximately $10 \mathrm{mTorr}$ and $1 \mathrm{~mL}$ of phosphoric acid $\left(\mathrm{H}_{3} \mathrm{PO}_{4}\right)$ is added. The vacutainer tubes are then heated to approximately $60{ }^{\circ} \mathrm{C}$ for 3-6 hours (if the sample is pure $\mathrm{CaCO}_{3}$, approximately $5.6 \mathrm{~mL}$ of $\mathrm{CO}_{2}$ is evolved at standard temperature and pressure). After the heat step, vacutainers are brought to atmospheric pressure either by venting excess $\mathrm{CO}_{2}$ or by adding sparged, triple-distilled water. 
Delivery of $\mathrm{CO}_{2}$ to the gas ion source is accomplished using the interface shown in Figure 2. Using a syringe pump operating at $0.5 \mathrm{~mL} / \mathrm{min}$, sparged, triple-distilled water is introduced into the vacutainer via a syringe needle. Positively displaced $\mathrm{CO}_{2}$ exits the vacutainer through a second syringe needle. At present, both syringe needles are manually inserted into the vacutainer. Water vapor is removed as the $\mathrm{CO}_{2}$ passes through a Nafion dryer (the necessity of the Nafion dryer, however, is currently under examination and recent results indicate that it is not absolutely necessary and that in fact it might cause a several per mille cross talk between samples). An open split is used to maintain a constant pressure of $\mathrm{CO}_{2}$ at the inlet of the capillary going to the ion source. Between samples, argon is used to back flush both the sample line and open split. In operation, care must be taken so the vacutainer doesn't overfill with water and cause water to enter the system and ion source.

A 'gas-bench' run on the ion source consists of a measurement of a suite of vacutainers; some vacutainers contain 'standard gas’ (i.e., gas of a known activity), some contain ‘dead-gas' (for instance $\mathrm{CO}_{2}$ generated from the IAEA C-1 reference material), some contain $\mathrm{CO}_{2}$ from secondary standards (for instance IAEA C-2 reference material), and of course some contain $\mathrm{CO}_{2}$ from the unknowns. An example of a run sequence with six unknowns is shown in Figure 3. In this run, measured blank values of $\mathrm{F}_{\mathrm{m}} \approx 5 \%$ were obtained (corresponding to a Libby age of greater than 42 kyrs before present). $F_{m}$ values of the same material as measured by conventional AMS (i.e., graphite) were between 2 and 3\%. For a modern sample, a statistical precision of 3 to 4\%o could be obtained in 5 to 6 minutes. However, reported measurement precision for a modern sample is about 6\%o and is controlled by the overall stability of the gas ion source and 
repeatability of the reference gas measurements. Nonetheless, the measurements are sufficiently precise for reconnaissance studies and for the selection of samples for further high-precision analyses using graphite-based techniques. In practice, typical throughput for the system is about 60 unknowns per day.

\section{Gas Chromatography}

As a scientific proof-of-principle, we have also coupled a gas-chromatograph to the ion source. In our setup, a gas chromatograph fitted with a megabore capillary column is used (small diameter capillary columns are precluded because of the large (microgram) samples required for AMS). The challenge is to match the high-flow output of the chromatograph $(\sim 8 \mathrm{~mL} / \mathrm{min}$ of carrier gas and eluent) with the flow requirements of the gas ion source $\left(\sim 370 \mu \mathrm{L} / \mathrm{min}\right.$ of $\mathrm{CO}_{2}$ plus argon carrier gas). Figure 4 is a schematic of how this was accomplished. Instead of the typical helium, hydrogen is used as the chromatographic carrier gas. After exiting the chromatographic column, the carrier gas and eluent are mixed with oxygen and argon (at 0.2 to $1.0 \mathrm{~mL} / \mathrm{min}$ ) before flowing into a combustion furnace. In the furnace, the eluent and hydrogen carrier gas are combusted to $\mathrm{CO}_{2}$ and $\mathrm{H}_{2} \mathrm{O}$ respectively. The Argon is unaffected by the combustion step and is used as a carrier gas to sweep the $\mathrm{CO}_{2}$ to the ion source. Water is removed with a Nafion dryer. An open split serves to maintain a constant pressure of $\mathrm{CO}_{2}$ and Argon at the inlet of the capillary going to the ion source. The open split was also fitted with an additional capillary that could be actuated in/out of the gas stream to supply reference $\mathrm{CO}_{2}$ to the ion source for normalization purposes. Further details of the setup and some initial test results can be found elsewhere ${ }^{[6,7]}$. 
An example ${ }^{14} \mathrm{C}$ chromatogram is shown in Figure 5. In this case, the sample introduced into the gas chromatograph was a mixture of biodiesel, a petroleum derived (i.e., 'dead') alkane, and two 'modern' fatty acid methyl esters. As can be seen, measured $F_{m}$ values are consistent with expected results. The precision on $\mathrm{F}_{\mathrm{m}}$ varied from $6-11 \%$ and was largely statistically limited. If higher precision had been desired, multiple injections could have been made and the run results combined. One can also see that the chromatogram is quite 'clean' with very little background between peaks. Although not shown here, chromatographic peaks containing as little as $2 \mu$ g of carbon have been measured. As can be seen when comparing Figures 3 and 5, measured $\mathrm{C}^{+}$ion currents are much lower in this case. This is because the gas flowing to the ion source is a mixture of Ar carrier gas and $\mathrm{CO}_{2}$. In general, there is a rough (but not perfect) linear relationship between $\mathrm{CO}_{2}$ concentration in the gas stream and resultant $\mathrm{C}^{+}$current. For example, if the ion source is set-up and tuned on a pure $\mathrm{CO}_{2}$ stream, the introduction of a 50/50 mixture of $\mathrm{Ar} / \mathrm{CO}_{2}$ results in $\mathrm{C}^{+}$currents of about $40 \%$ of the pure $\mathrm{CO}_{2}$ case (as opposed to the expected $50 \%)$.

\section{Summary}

We have developed a novel, low-memory, gas-accepting ion source that produces ion currents typical of a traditional graphite source. A key advantage of a gas source is that, since the graphitization step in sample processing is eliminated, the total time required for sample analysis is shortened and the potential for sample-handling induced contamination is reduced. A key shortcoming of the gas ion source is its relatively low efficiency. With a $\mathrm{CO}_{2}$ to $\mathrm{C}^{-}$efficiency of less than $1 \%$, the present source is ill suited for small samples and/or measurements requiring high precision. 
We have coupled both a 'gas-bench' device and gas-chromatograph to the ion source. With the gas bench we have been able to provide radiocarbon analysis of carbonates with results sufficiently precise for reconnaissance studies where large numbers of samples need to be analyzed quickly at low-cost. With the gas chromatograph interface, we have demonstrated realtime, compound-specific ${ }^{14} \mathrm{C}$ analysis of a complex mixture at natural abundance levels. This represents a significant breakthrough in ${ }^{14} \mathrm{C}$ analysis and should prove useful for a variety of environmental and biomedical applications.

Finally, the characteristics of the ion source make it amenable to interfacing with a variety of other analytical instrumentation. Other possible interfaces include an elemental analyzer for organic samples, a laser-combustion system for spatially resolved ${ }^{14} \mathrm{C}$ analysis, and a high performance liquid-chromatograph.

\section{Acknowledgment}

This work is supported by a Cooperative Agreement (OCE- 2310753487) with the US National Science Foundation. 


\section{References}

[1] J.S. Vogel, J.R. Southon, D.E. Nelson, and T.A. Brown, Performance of catalytically condensed carbon for use in accelerator mass spectrometry, Nuclear Instruments and Methods in Physics Research Section B, Volume 5, Issue 2, November 1984, Pages 289293.

[2] C. Bronk Ramsey, P. Ditchfield, and M. Humm, Using a gas ion source for radiocarbon AMS and GC-AMS. Radiocarbon, Volume 46, Number 1, 2004, Pages 25-32.

[3] M.L. Roberts, K.F. von Reden, C.P. McIntyre, and J.R. Burton, Progress with a gasaccepting ion source for Accelerator Mass Spectrometry, Nuclear Instruments and Methods in Physics Research Section B: Beam Interactions with Materials and Atoms (2011) doi:10.1016/j.nimb.2011.04.017.

[4] B.X. Han, K.F. von Reden, M.L. Roberts, R.J. Schneider, J.M. Hayes, and W.J. Jenkins, Electromagnetic field modeling and ion optics calculations for a continuous-flow AMS system, Nuclear Instruments and Methods in Physics Research Section B: Beam Interactions with Materials and Atoms, Volume 259, Issue 1, 2007, Pages 111-117.

[5] K.F. von Reden, M.L. Roberts, C.P. McIntyre, and J.R. Burton, Design and reality: Continuous-flow accelerator mass spectrometry (CFAMS), Nuclear Instruments and Methods in Physics Research Section B: Beam Interactions with Materials and Atoms (2011) doi:10.1016/j.nimb.2011.04.019.

[6] C.P. McIntyre, S.P. Sylva, and M.L. Roberts, Gas Chromatograph-Combustion System for C-14-Accelerator Mass Spectrometry, Analytical Chemistry, Volume 81, Issue 15, 2009, Pages 6422-6428. 
[7] C.P. McIntyre, E. Galutschek, M.L. Roberts, K.F. von Reden, A.P. McNichol, and W.J. Jenkins, A continuous-flow gas chromatography ${ }^{14} \mathrm{C}$ accelerator mass spectrometry system, Radiocarbon, Volume 52, Numbers 2-3, 2010, Pages 295-300. 
Table I. Typical ion source output and transmission efficiencies.

Gas Flow $\left(\mathrm{CO}^{2}\right)$

$370 \mu \mathrm{l} / \mathrm{min}$

Low-Energy ${ }^{12} \mathrm{C}^{-}$

$100-150 \mu \mathrm{A}$

High-Energy ${ }^{12} \mathrm{C}^{+}$

$30-40 \mu \mathrm{A}$

${ }^{14} \mathrm{C}$ count Rate (from $\mathrm{F}_{\mathrm{m}}=1.0438 \mathrm{CO}_{2}$ )

$\sim 350$ cps

Ion Source Efficiency $\left(\mathrm{C}^{+} /\right.$Molecule $\left.\mathrm{CO}_{2}\right)$

$>6.3 \%$

Charge Exchange Canal Efficiency $\left(\mathrm{C}^{+}\right.$to $\left.\mathrm{C}^{-}\right)$

$12 \%$

Accelerator Transmission Efficiency $\left(\mathrm{C}^{-}\right.$to $\left.\mathrm{C}^{+}\right)$

$29 \%$

Percent time in one bouncing cycle spent on ${ }^{14} \mathrm{C}$

$97.6 \%$

Total System Efficiency

$0.21 \%$ 


\section{Figure Captions}

Figure 1. Operating principle of the gas ion source. $\mathrm{CO}_{2}$ is disassociated in the plasma chamber and $\mathrm{C}^{+}$ions extracted. Negative ions are formed by charge-exchanging interactions with magnesium.

Figure 2. A schematic of the gas bench interface. $\mathrm{CO}_{2}$ is positively displaced from vacutainers into the gas ion source.

Figure 3. A run sequence, containing six unknowns, using the 'gas-bench'. The measured $\mathrm{F}_{\mathrm{m}}$ and uncertainty (in parentheses) is shown for each sample. Ref stands for reference gas and has a known $\mathrm{F}_{\mathrm{m}}=1.044$. $\mathrm{C}-1$ is one of the IAEA standards and has a consensus $\mathrm{F}_{\mathrm{m}}<0.001$.

Figure 4. Gas chromatograph to ion source interface.

Figure 5. ${ }^{14} \mathrm{C}$ Gas chromatographic analysis of mixture of compounds. The $\mathrm{nC} 16$ alkane is petroleum derived. Fatty acid methyl esters (FAME) C15 and C17 are derived from 'modern' sources. The biodiesel was purchased locally. The approximate mass of each compound is indicated below its name. STD indicates injection of reference gas $\left(F_{m}=1.0438\right)$ used for normalization. $F_{m}$ values for unknowns are given on the ${ }^{14} \mathrm{C}$ trace. As expected, the biodiesel has $\mathrm{F}_{\mathrm{m}}$ values consistent with current ${ }^{14} \mathrm{C}$ atmospheric levels. $\mathrm{C}^{+}$ion currents are lower than in the gas bench example because in this case the gas flowing to the ion source is a mixture of $\mathrm{CO}_{2}$ and $\mathrm{Ar}$ carrier gas. 


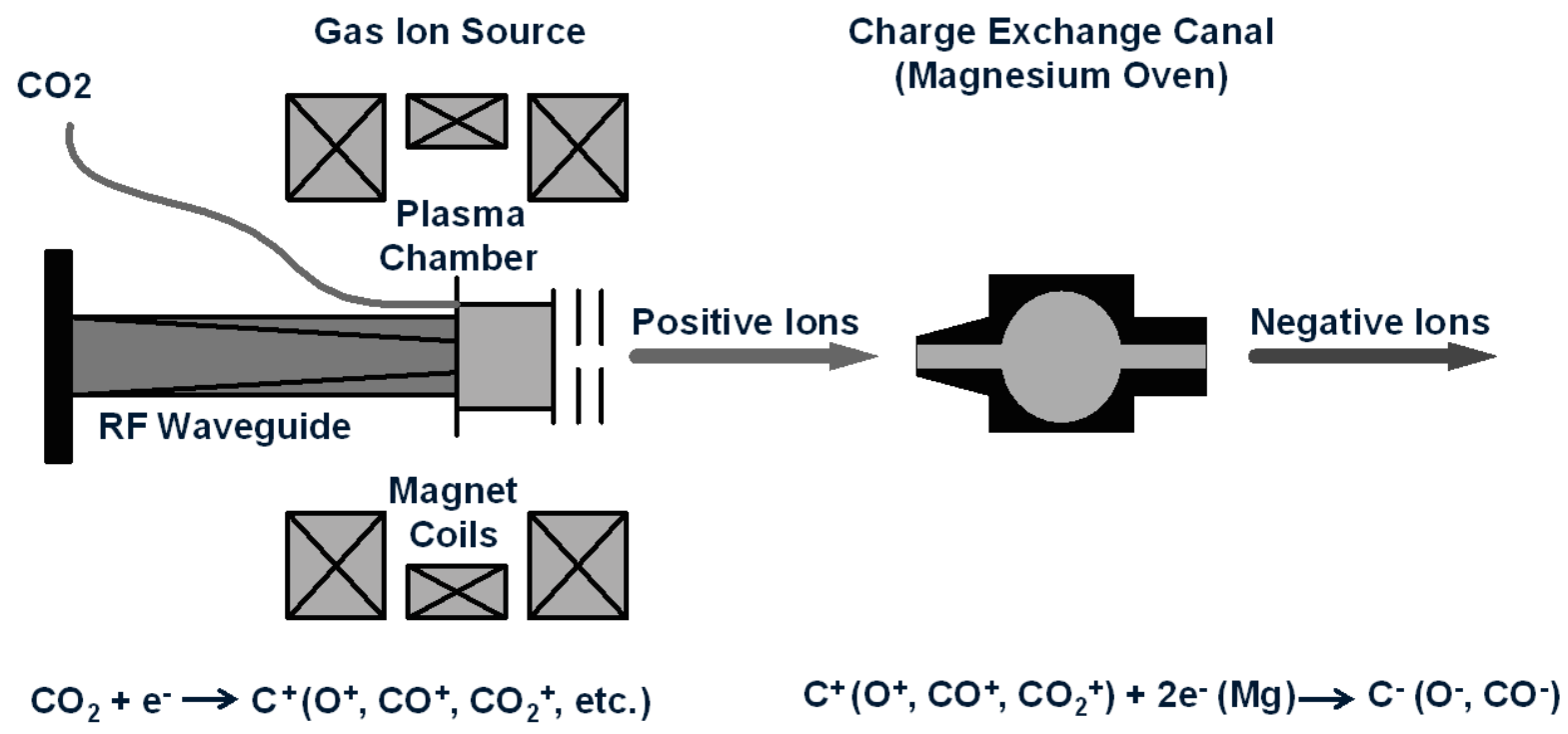

Figure 1 


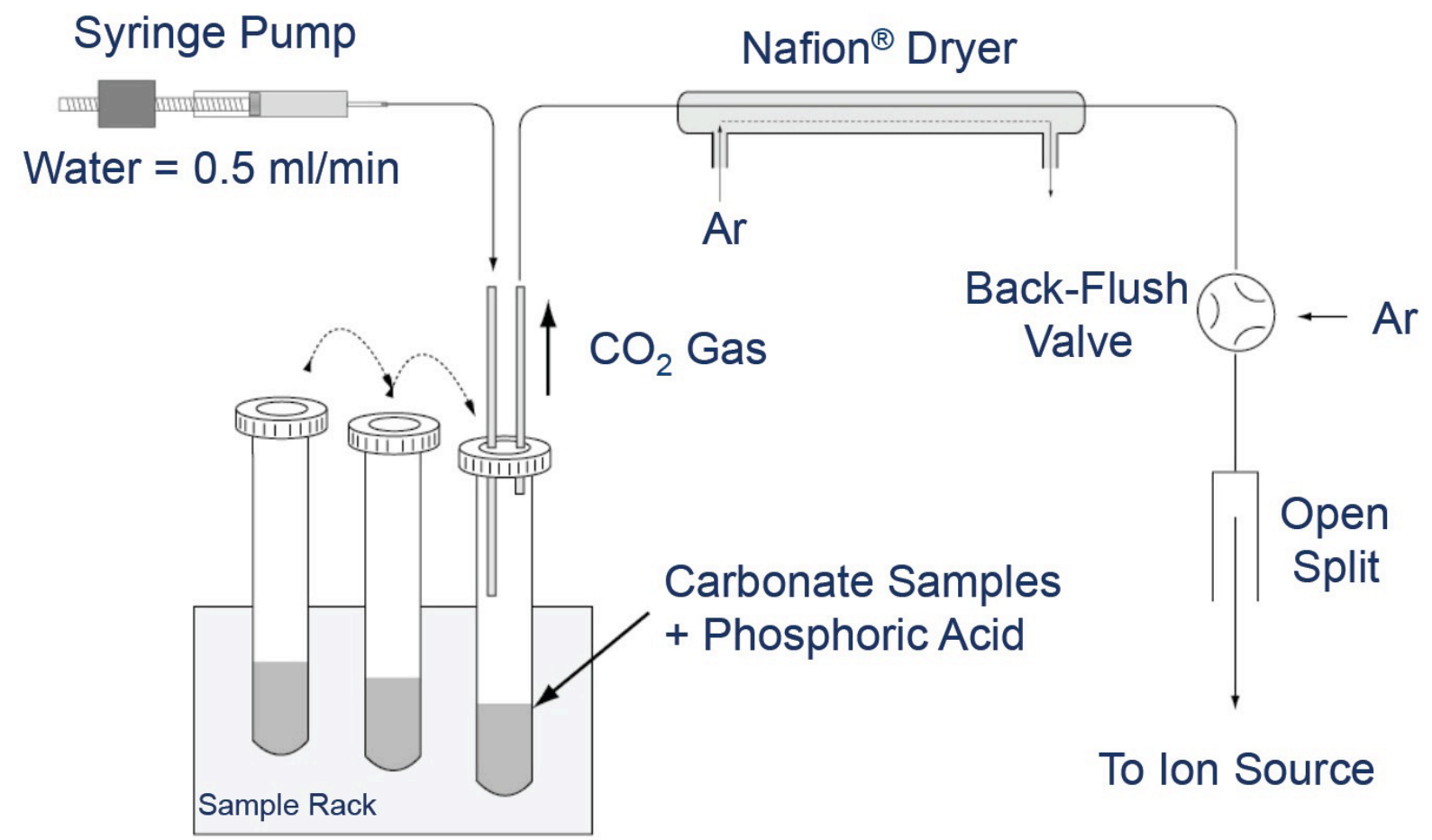

Figure 2 


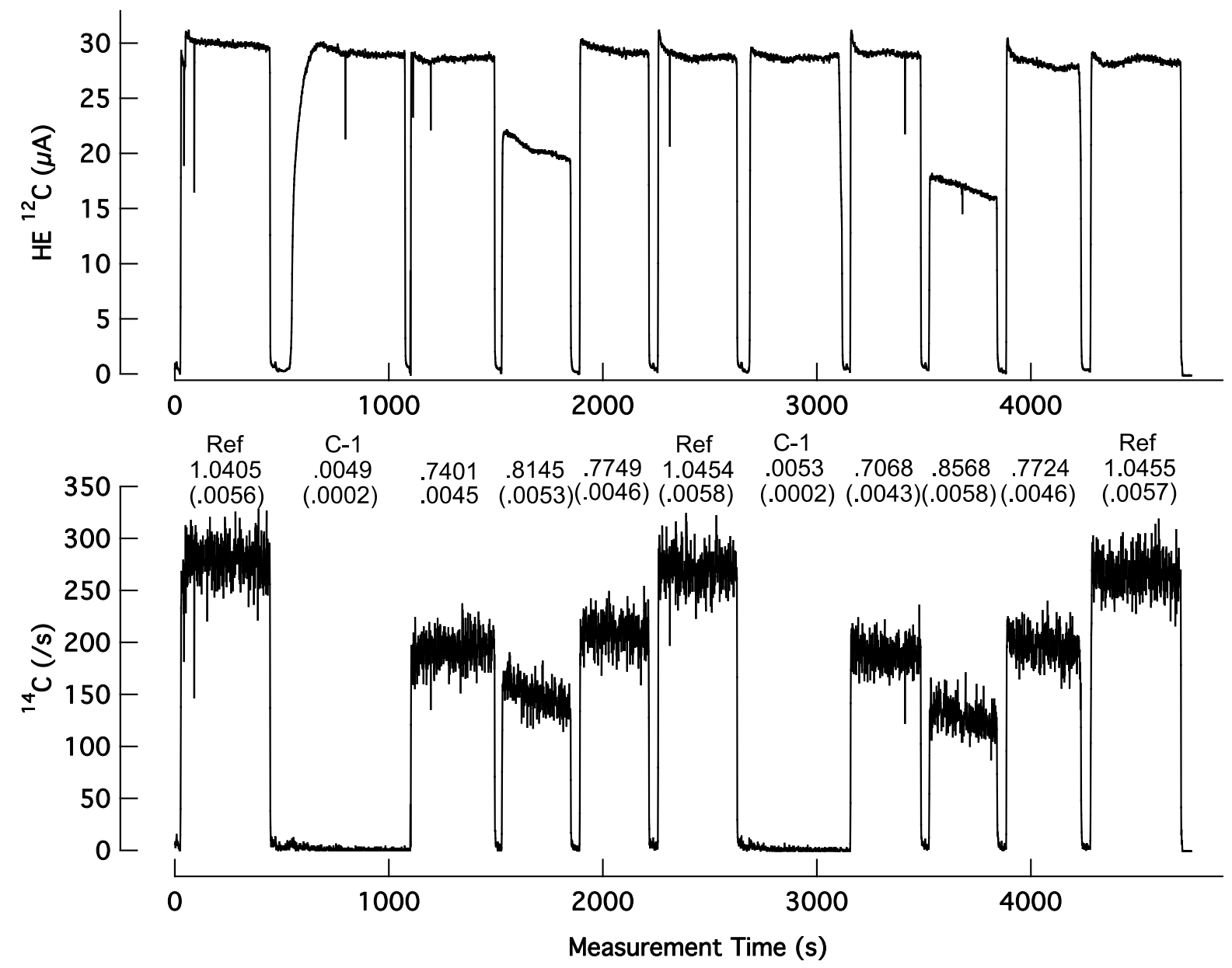

Figure 3 


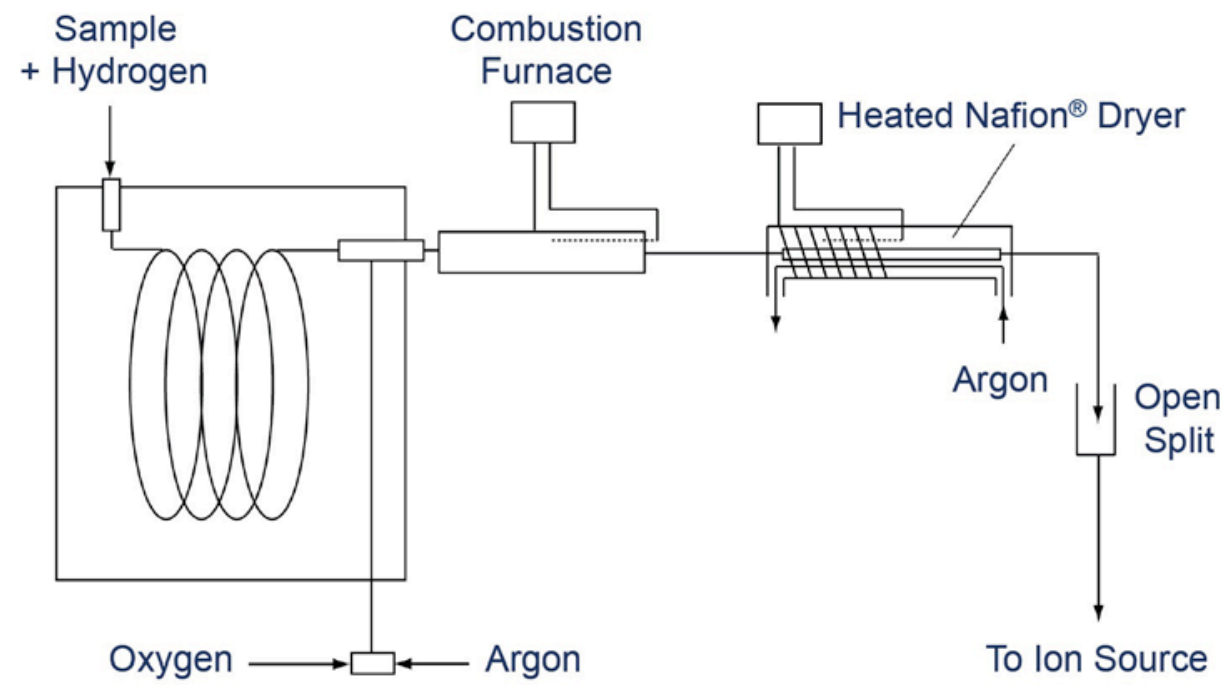

Figure 4 

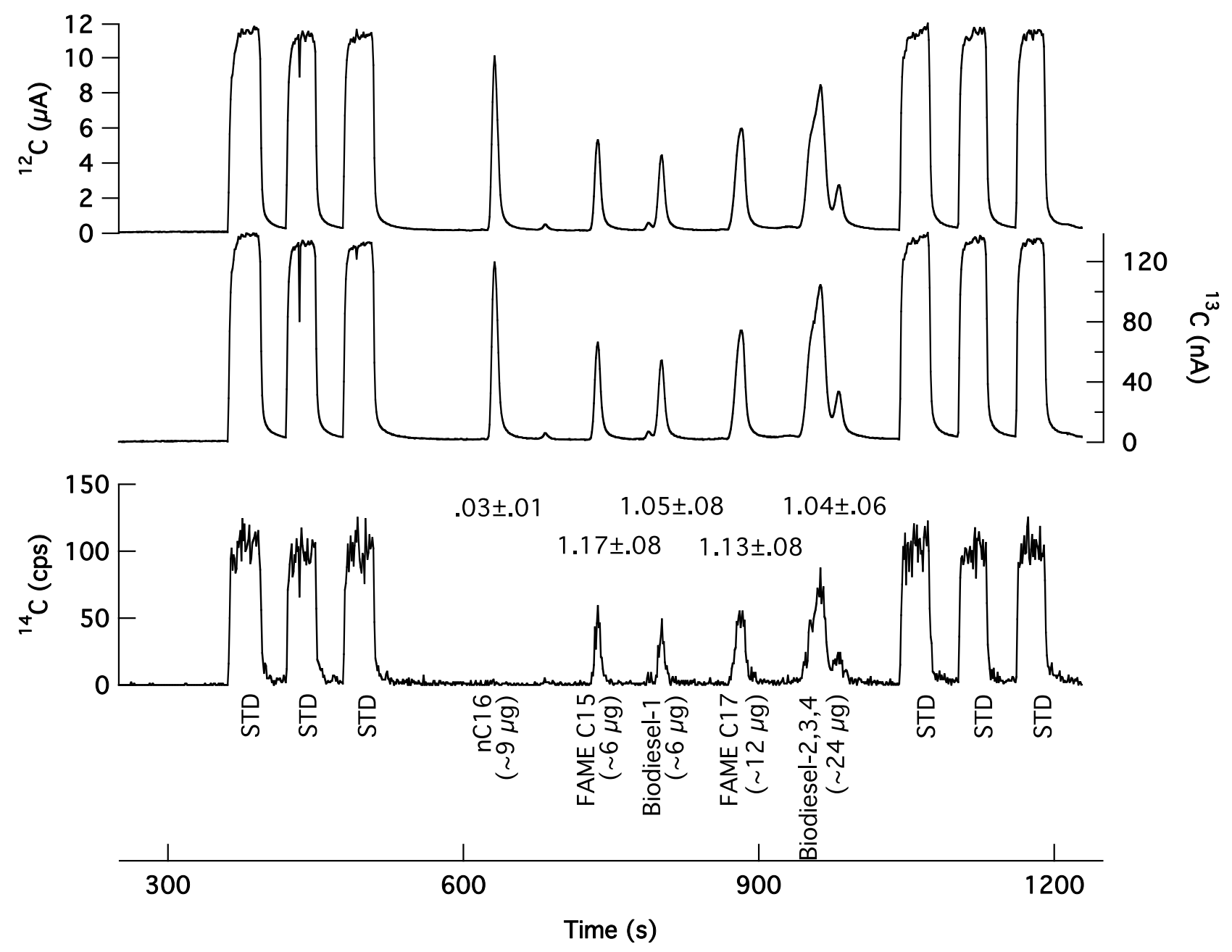

Figure 5 